\title{
Odontoclínica: simulação de gestão em clínica odontológica em um curso de Graduação em Odontologia
}

Renato Magalhães Costa*; Neiana Carolina Rios Ribeiro*; Urbino da Rocha Tunes**; Marcelo Nunes Dourado Rocha $^{* * *}$; Maurício dos Santos Lago ${ }^{* * * *}$

\footnotetext{
* Graduação em Odontologia pela Escola Bahiana de Medicina e Saúde Pública (EBMSP)

** Coordenador do Curso de Graduação em Odontologia e Professor Titular de Periodontia da Escola Bahiana de Medicina e Saúde Pública (EBSMP)

*** Doutor em Saúde Coletiva pela Universidade Federal da Bahia (UFBA) e Professor Assistente de Saúde Coletiva da Escola Bahiana de Medicina e Saúde Pública (EBMSP)

**** Mestre em Odontologia e Professor Assistente de Clínica Integrada V (Endodontia) da Escola Bahiana de Medicina e Saúde Pública (EBMSP)
}

\section{RESUMO}

A constante entrada de cirurgiões-dentistas no mercado de trabalho ocasiona um crescimento de concorrência entre os profissionais.

Nesse cenário, é necessário apresentar diferenciais para se destacar, como habilidades em gestão empresarial. Saber administrar um consultório ou clínica é pré-requisito para alcançar sucesso e estabilidade na profissão. Apesar disso, a maioria dos cursos de graduação em odontologia não aborda essa temática na sua grade curricular. Esse trabalho tem como objetivo discutir o estudo de gestão empresarial de clínica odontológica em um curso de Graduação em Odontologia, através do relato de experiência da Odontoclínica, uma hipotética empresa sobre a responsabilidade da "Turma de Odontologia 2013-2 Prof. Antístenes Albernaz Alves Neto" da Escola Bahiana de Medicina e Saúde Pública (EBMSP) e parte do componente curricular Clínica Integrada V. Para a realização das atividades foi definida uma estrutura diretiva composta por presidente, dois superintendentes e quatro diretorias (técnica, administrativa, financeira e científica), além da criação de uma moeda fictícia (Bahianinos - BH\$). Durante os três meses de funcionamento da Odontoclínica foram realizados 2.180 procedimentos (entre procedimentos gerais e nas áreas de periodontia, endodontia, dentística e prótese dentária). Com a realização destes procedimentos foi arrecadado um montante de BH\$ 76.683,71 (custo total), tendo como receita prevista a quantia de $\mathrm{BH} \$ 38.341,85$. É essencial que os cursos de graduação em odontologia assumam postura mais ativa sobre gestão empresarial na formação dos novos profissionais. A estratégia de negócio, junto com um bem delineado plano operacional e financeiro, influencia no sucesso profissional e empresarial.

Descritores: Odontologia. Administração da Prática Odontológica. Mercado de Trabalho.

\section{INTRODUÇÃO}

O mercado de trabalho em odontologia na América Latina e no Brasil, segundo Matsubara $^{1}$, vem apresentando muitas mudanças nas últimas décadas. $\mathrm{O}$ ingresso de um grande número de novos profissionais e o 
aumento da atuação das empresas de convênios na área odontológica influenciaram substancialmente na estrutura do mercado.

Devido à grande concentração de consultórios odontológicos em grandes centros urbanos, ocorre a geração de um mercado de trabalho altamente competitivo nestes locais, o que leva os profissionais a enfrentar altos desafios para conquistar clientes ${ }^{2,3}$.

Zimbres $^{4}$ afirma que isso demanda por uma maior diferenciação dos serviços oferecidos para se obter vantagem competitiva. Tal diferenciação depende não somente dos aspectos técnicos, mas também de aspectos relacionados à gestão de um consultório odontológico, como análise financeira, definição do planejamento estratégico e administração de pessoal.

Apesar disso, os cirurgiões-dentistas geralmente não recebem nenhum treinamento nessa área nos cursos de graduação ${ }^{1}$.

Segundo pesquisa divulgada pelo Sebrae em $2004^{5}$, entre $70 \%$ e $80 \%$ das empresas no Brasil fecham as portas antes de completarem dois anos de vida. Os motivos são vários e os empresários se justificam dizendo que foi por causa da alta carga tributária ou por que a economia está ruim ou ainda a falta de apoio do governo. Pode-se encarar a situação por outra ótica: a falta de planejamento.

É cada vez maior o número de consultórios odontológicos que, diante da complexidade no cenário empresarial e de tantas turbulências e incertezas, estão buscando ferramentas e técnicas para que as auxiliem no processo gerencial ${ }^{5}$.

O serviço odontológico é um serviço que envolve alta complexidade, pois envolve vários tipos de materiais e fornecedores, profissionais especializados e equipe auxiliar. Esse conjunto de fatores, interagindo de maneira dinâmica e personalizada de acordo com a necessidade de cada paciente, aliado à grande competitividade existente no mercado moderno, desperta a necessidade do uso de conceitos de administração para melhor gerenciar um consultório odontológico ${ }^{6}$.

O sucesso de consultório odontológico está relacionado a dois fatores primordiais: planejamento estratégico de qualidade e capacidade em gestão administrativa ${ }^{7}$.

O desafio de se encaixar no mercado é maior para os recém-formados porque eles não têm experiência e nem clientela cativa. Para os profissionais que estão adentrando no mercado, existem três opções: trabalhar na saúde pública, em consultórios de terceiros ou em consultórios próprios. Caso o profissional decida iniciar um serviço próprio, necessitará de conhecimentos sobre organização e orientação estratégica ${ }^{1}$.

A resolução CNE/CES 3, de 19 de fevereiro de 2002, traz as Diretrizes Curriculares Nacionais do Curso de Graduação em Odontologia $^{8}$. No artigo 4, inciso V, está declarado que a formação do Cirurgiãodentista tem por objetivo dotar o profissional dos conhecimentos requeridos para o exercício de competências e habilidades gerais. Sobre administração e gerenciamento, preconiza-se que os profissionais devem estar aptos a tomar iniciativas, fazer o gerenciamento e administração da força de trabalho, dos recursos físicos e materiais e de informação, da mesma forma que devem estar aptos a serem empreendedores, gestores, empregadores ou lideranças na equipe de saúde.

Esse estudo tem como objetivo discutir gestão empresarial de clínica odontológica em um curso de graduação em odontologia, através do relato de experiência da Odontoclínica, uma hipotética empresa sobre a responsabilidade da "Turma de Odontologia 2013-2 Prof. Antístenes Albernaz Alves Neto" da Escola Bahiana de Medicina e Saúde Pública (EBMSP) e parte do componente curricular Clínica Integrada V. 


\section{RELATO DE EXPERIÊNCIA}

A Odontoclínica consistiu na simulação de uma clínica odontológica realizada pelos discentes da "Turma de Odontologia 2013.2 Prof. Antístenes Albernaz Alves Neto" (turno matutino e vespertino), da Escola Bahiana de Medicina e Saúde Pública (EBMSP), no período de agosto a novembro de 2012. Como os valores de transação monetária foram hipotéticos, foi criada uma moeda fictícia para quantificar esses valores, denominada Bahianinos (BH\$).

Para a realização das atividades foi definida uma estrutura diretiva composta por Presidente, dois Superintendentes e quatro Diretorias (Técnica, Administrativa, Financeira e Científica), todas contendo sócios do turno matutino e vespertino. A presidência tinha a função de coordenar e orientar os demais setores, liderando as atividades. Superintendentes deveriam supervisionar as atividades, garantindo que todos os grupos funcionem em conjunto, havendo boa comunicação entre as diretorias.

A diretoria técnica foi responsável pelo acompanhamento, controle e avaliação dos resultados apresentados em relação aos objetivos esperados. A diretoria administrativa foi incumbida dos poderes e atribuições de administrar, gerando balancetes e analisando através de gráficos estatísticos evolutivos e comparativos toda produção da pseudoclínica. A diretoria financeira foi encarregada de elaborar um planejamento financeiro, para assim conseguir manter a empresa em funcionamento. E, por fim, a diretoria científica foi responsável com elaboração de projetos e artigos para posterior divulgação da Odontoclínica, como o presente trabalho.

Para formação da Odontoclínica foi imprescindível seguir um planejamento estratégico constituído por algumas etapas: contrato social, plano operacional e um plano financeiro.
Foi elaborado um contrato social fictício adotando os pré-requisitos e encargos sociais para a criação de uma pessoa jurídica, com existência e patrimônio distintos da pessoa física dos sócios.

A atividade empresarial foi exercida pela sociedade representada por seus administradores. O capital social contava com BH\$ 362.500,00 Bahianinos, dividido em 362.500 quotas de valor nominal $\mathrm{BH} \$ 1,00$, integralizadas pelos 46 sócios (os discentes cursando o componente curricular Clínica Integrada V). Portanto, cada sócio possuía 7.880 quotas (BH\$ 7.800,000 Bahianinos).

Como definido no plano operacional, a Odontoclínica funcionava em dois dias úteis da semana, com um total de 46 cirurgiões dentistas. Cada dentista tinha 02 (dois) turnos de trabalho, com carga horária semanal de 8 horas.

A estrutura básica era composta pela sala de espera, sanitários, consultórios, sala de aplicações com aparelhos, escritório/administração e o depósito. Os itens Básicos eram: móveis em geral, sistema telefônico, sistema de ar condicionado, sistema de música ambiente; computadores, softwares gerenciais, impressoras, materiais de escritório, consultórios, matérias odontológicos, produtos de higienização, uniformes, equipamentos odontológicos (autoclave, estufa, amalgamador, fotopolimerizador, ultrassom, compressor, bomba a vácuo, aparelho de raios $\mathrm{X}$ odontológico, entre outros) e materiais descartáveis.

A Odontoclínica contava ainda com a presença de duas Auxiliares de Saúde Bucal, um Técnico de Manutenção de Equipamentos, um Auxiliar de Serviços Gerais, um Técnico em Prótese Dentária e uma Recepcionista, que trabalharam em dois turnos de $4 \mathrm{~h} \mathrm{du}-$ rante dois dias úteis da semana, totalizando carga horária de $16 \mathrm{~h}$ semanais (64 horas mensais). A carga horária de trabalho repre- 
sentava a carga horária de atendimento na Clínica Integrada V (atendimentos em dois dias na semana, com uma turma matutina e outra vespertina, em ambos os dias).

Para executar o Plano Financeiro foi realizado um levantamento dos recursos para investimento na empresa, o que constitui o Investimento Total. O Investimento Total é composto pelos investimentos fixos, capital de giro e investimentos pré-operacionais.

Os investimentos fixos correspondem a todos os bens que devem ser comprados para que o negócio possa funcionar de maneira apropriada, como cadeiras odontológicas, aparelhos de Raios-X Odontológico, compressores e fotopolimerizadores. A partir do inventário feito, os elementos de investimento fixos disponíveis somaram BH\$ 298.890,00. O total da estimativa de investimento inicial foi BH\$ 93.000,00, sendo que o material de consumo foi responsável por

Para a abertura da Odontoclínica, com a montagem de 30 cadeiras odontológicas para 46 sócios, foi necessário um investimen-
$\mathrm{BH} \$ 11.000,00$ e os instrumentais por $\mathrm{BH} \$$ $82.000,00$.

O Capital de Giro é o montante de recursos necessário para o funcionamento normal da empresa, compreendendo a compra de matérias-primas ou mercadorias, financiamento das vendas e o pagamento das despesas. Para estimar o capital de giro para o começo das atividades da empresa, foi apurado o estoque inicial e o caixa mínimo necessário. O total do capital de giro foi $\mathrm{BH} \$$ 22.205,00. O estoque inicial foi BH\$ $11.000,00$ e o total mínimo do caixa era BH\$ 11.205,00.

Além do investimento inicial com a estrutura e materiais, para que uma empresa possa iniciar suas atividades é necessário que esteja devidamente legalizada, ou seja, deverá estar registrada em determinados órgãos nos âmbitos federal, estadual e municipal, estes estão descritos na Tabela 1.

to total de BH\$ 415.059,70. Todos os gastos são expostos em resumo na Tabela 2.

Tabela 1 - Descrição de impostos para abertura da empresa

\begin{tabular}{lcc}
\hline \multicolumn{1}{c}{ IMPOSTO } & Natureza & Valor (BH\$) \\
\hline SUCOM (Superintedência de Controle e Ordenamento do & Municipal & 379,90 \\
Solo do Município) & Estadual & 159,00 \\
TFF (Taxa de Fiscalização de Funcionamento) & Federal & 10,00 \\
JUCEB (Junta Comercial do Estado da Bahia) & Municipal & 415,80 \\
CNPJ (Cadastro Nacional da Pessoa Jurídica) & Total & $\mathbf{9 6 4 , 7 0}$ \\
\hline
\end{tabular}

Para controle da Odontoclínica foram desenvolvidos formulários padronizados nos quais os discentes registravam, após o atendimento de cada paciente, a descrição do procedimento, quantidade, código do SUS correspondente ao procedimento, custo unitário/sessão, custo total e receita prevista.
O custo unitário era verificado em tabelas disponibilizadas pelos docentes do componente curricular. Constava nessas tabelas os materiais utilizados e o custo da sessão para a realização de cada procedimento de prótese dentária, endodontia, dentística e periodontia que eram realizados na clínica. 
Para calcular o custo total (montante arrecadado), o discente deveria utilizar a seguinte fórmula: custo unitário/sessão x quantidade de procedimentos x 2 (margem de lucro). A receita prevista por sua vez era a metade do custo total.

Durante os três meses de funcionamento da Odontoclínica foram realizados 1.163 procedimentos gerais, 323 procedi- mentos periodontais, 130 procedimentos de endodontia, 309 de dentística e 255 de prótese dentária. Com a realização destes procedimentos foi arrecadado um montante de BH\$ 76.683,71 (custo total), tendo como receita prevista a quantia de $\mathrm{BH} \$ 38.341,85$. A tabela 3 resume o custo total e receita prevista por especialidade odontológica.

Tabela 2 - Descrição do investimento total

\begin{tabular}{lc}
\hline \multicolumn{1}{c}{ Descrição } & Valor (BH\$) \\
\hline Montagem da clínica e aparelhos & $298.890,00$ \\
Instrumentais & $82.000,00$ \\
Estoque inicial de material de consumo & $11.000,00$ \\
Capital de Giro & $22.205,00$ \\
Impostos para abertura & 964,70 \\
\hline & Total para Investimento \\
\hline & Total por sócio (46) \\
\hline
\end{tabular}

Contudo, como toda empresa, a lucro da empresa foi BH\$ 35.838,11. DividiOdontoclínica gerou gastos mensais com do entre os 46 sócios, o lucro de cada um foi funcionários, impostos, manutenção da estru- $\mathrm{BH} \$$ 779,08. A descrição dos gastos gerais, tura física, entre outros. O total de gastos folha de pagamento dos funcionários, impospara o funcionamento da empresa no trimes- tos mensais, impostos trimestrais e simulação tre somou o valor de BH\$ 40.845,60. Dimi- do lucro constam, respectivamente, nas tabenuído esse valor do custo total arrecadado, o las 4 a 8.

Tabela 3 - Custo total e receita prevista em todos os procedimentos (gerais, periodontia, endodontia, dentística e prótese)

\begin{tabular}{|c|c|c|}
\hline Procedimentos & Custo total (BH\$) & $\begin{array}{c}\text { Receita prevista } \\
\text { (BH\$) }\end{array}$ \\
\hline Gerais & $8.414,16$ & $4.207,08$ \\
\hline Periodontia & $13.159,34$ & $6.579,67$ \\
\hline Endodontia & $13.653,44$ & $6.826,72$ \\
\hline Dentística & $18.188,18$ & $9.094,09$ \\
\hline Prótese Dentária & 23.268,59 & $12.255,73$ \\
\hline TOTAL & $76.683,71$ & $38.341,85$ \\
\hline
\end{tabular}


Tabela 4 - Descrição dos gastos gerais

\begin{tabular}{lc}
\hline \multicolumn{1}{c}{ Descrição } & Valor (BH\$) \\
\hline Luz & 552,00 \\
Água & 245,33 \\
Espaço físico (Estacionamento, manutenção, limpeza) & $1.581,00$ \\
Telefone e Internet & 876,66 \\
Gastos Gerais Mensais & $\mathbf{3 . 2 5 4 . 9 9}$ \\
Gastos gerais no trimestre & $\mathbf{9 . 7 6 4 , 9 7}$ \\
Gastos com materiais de consumo no trimestre & $\mathbf{1 2 . 7 9 4 , 7 2}$ \\
\hline
\end{tabular}

Tabela 5 - Folha de pagamento dos funcionários

\begin{tabular}{|c|c|}
\hline Descrição & Valor (BH\$) \\
\hline Salário base & 360,00 \\
\hline FGTS (8\%) & 28,00 \\
\hline INSS $(8 \%) *$ & 28,00 \\
\hline Férias proporcionais & 90,00 \\
\hline $13^{\circ}$ salário proporcional & 90,00 \\
\hline Subtotal no trimestre por funcionário & $1.344,00$ \\
\hline Total no trimestre para os 06 funcionários & $8.064,00$ \\
\hline
\end{tabular}

*O INSS é um imposto descontado na folha de pagamento do funcionário e não da empresa.

Tabela 6 - Impostos mensais

\begin{tabular}{lcc}
\hline & Descrição & Valor (BH\$) \\
\hline PIS (0,65\%) & 166,14 \\
COFINS (3\%) & & 766,83 \\
ISS (2\%) & & 511,22 \\
Subtotal mensal & & $\mathbf{1 . 4 4 4 , 1 9}$ \\
Total no trimestre & & $\mathbf{4 . 3 3 2 , 5 7}$ \\
\hline
\end{tabular}

Tabela 7 - Impostos trimestrais

\begin{tabular}{lcc}
\hline & Descrição & Valor (BH\$) \\
\hline IRPJ (15\%)* & & $3.680,82$ \\
CSLL (9\%) & & $2.208,49$ \\
Total no trimestre & & $\mathbf{5 8 8 9 , 3 1}$ \\
\hline
\end{tabular}

*O IRPJ é calculado em cima de 32\% do valor total arrecadado

Tabela 8 - Simulação do lucro obtido se fossem utilizadas diferentes margens de lucro

\begin{tabular}{cccccc}
\hline $\begin{array}{c}\text { Custos com proce- } \\
\text { dimentos (BH\$) }\end{array}$ & $\begin{array}{c}\text { Valor } \\
\text { cobrado } \\
\text { (BH\$) }\end{array}$ & $\begin{array}{c}\text { Montante ar- } \\
\text { recadado } \\
\text { (BH\$) }\end{array}$ & $\begin{array}{c}\text { Total gastos } \\
\text { (BH\$) }\end{array}$ & $\begin{array}{c}\text { Total lucro } \\
\text { (BH\$) }\end{array}$ & $\begin{array}{c}\text { Lucro por } \\
\text { sócio (BH\$) }\end{array}$ \\
\hline $8.341,85$ & $2 \mathrm{x}$ & $76.683,70$ & $40.845,60$ & $35.838,10$ & $\mathbf{7 7 9 , 0 8}$ \\
$38.341,85$ & $3 \mathrm{x}$ & $115.025,55$ & $65.993,99$ & $49.031,56$ & $\mathbf{1 0 6 5 . 9 0}$ \\
$38.341,85$ & $4 \mathrm{x}$ & $153.367,40$ & $77.784,12$ & $75.583,28$ & $\mathbf{1 . 6 4 3 , 1 1}$ \\
$38.341,85$ & $5 \mathrm{x}$ & 191.709 .25 & $89.574,26$ & $102.134,99$ & $\mathbf{2 . 2 2 0 , 3 2}$ \\
$38.341,85$ & $10 \mathrm{x}$ & 383418,50 & $148.524,86$ & $234.893,64$ & $\mathbf{5 . 1 0 6 . 3 8}$ \\
\hline
\end{tabular}




\section{DISCUSSÃO}

Andrade et al. ${ }^{9}$ afirmam que grande parte dos profissionais da Odontologia tentam iniciar o seu próprio negócio, mas na maioria das vezes as universidades não disponibilizam na graduação matéria específica que trate do empreendedorismo.

A economia mundial opera com novos padrões de relações de trabalho, onde a estabilidade profissional se posiciona longe da realidade encontrada no mercado. Isso induz a repensar a cultura de formação das Instituições de Ensino Superior que formam o profissional do futuro. Os desafios dizem respeito não só às mudanças de metodologia pedagógica, mas também à conscientização do corpo discente e do corpo docente de que o melhor método de ensino a ser adotado pressupõe o comprometimento e a integração do corpo acadêmico ${ }^{10}$.

A Odontoclínica não foi desenvolvida com a pretensão de capacitar os discentes na complexa função de gestão em clínica odontológica, mas sim, despertar neles a percepção da necessidade de conhecimento sobre a área. As habilidades desenvolvidas na faculdade, tanto as científicas quanto as técnicas, não são suficientes para um profissional obter sucesso ao trabalhar como gestor de sua empresa privada.

A dificuldade de encontrar referencial teórico em trabalhos publicados em bons periódicos reforça a ausência de atenção que o assunto gestão de clínica odontológica sofre.

A Odontologia é uma das áreas nas quais os profissionais em geral não possuem um conhecimento adequado do exercício de empreender e de seus impactos. Os conceitos da administração são pouco explorados, o que dificulta o entendimento da dinâmica do mercado e as oportunidades e ameaças desse nicho $^{11}$. O empreendedorismo tem se mostrado um grande aliado do desenvolvimento econômico, pois tem dado suporte à maioria das inovações que têm promovido esse desenvolvimento ${ }^{12}$.

Gerir, hoje, envolve uma gama muito mais abrangente e diversificada de atividades do que no passado. A evolução das organizações em termos de modelos estruturais e tecnológicos, tendo as mudanças e o conhecimento como novos paradigmas, tem exigido uma nova postura nos estilos pessoais e gerenciais voltados para uma realidade diferenciada e emergente. Conseqüentemente o gestor hoje precisa estar apto a perceber, refletir, decidir e agir em condições totalmente diferentes das de antes ${ }^{13}$.

O profissional da Odontologia deve gerir seu consultório levando em conta que se trata de uma pequena empresa e seu comportamento empreendedor é de vital importância para que este quebre as barreiras impostas pelo mercado em geral ${ }^{14}$.

O lucro da Odontoclínica foi BH\$ 38.742,51. A margem de lucro dessa empresa fictícia foi o dobro do custo por sessão. Essa margem de lucro facilitava os cálculos que deviam ser realizados após o atendimento de cada paciente, mas não reflete a margem de lucro de empresas odontológicas reais. Por exemplo, nos procedimentos protéticos, o total arrecadado pode variar entre 4 e 6 vezes o custo laboratorial. Em endodontia, a margem de lucro pode ser até 10 vezes maior que o custo dos materiais utilizados.

O lucro obtido durante o período analisado não foi satisfatório, e vale ressaltar que a estratégia da empresa é de suma importância para determinar valores compatíveis com o público alvo para que possam ser obtidos valores de lucro mais favoráveis. O lucro é importante para remuneração do capital investido, reinvestimento no próprio consultório e remuneração dos sócios, ou seja, é precisamos contemplá-lo na formação do preço 
de forma justa para que o próprio empreendimento consiga sobreviver no mercado ${ }^{15}$.

Na Tabela 8, há a simulação do lucro obtido se outras margens de lucro fossem adotadas. Com a alteração da margem de lucro, seria obtido um retorno financeiro mais satisfatório e mais próximo da realidade dos profissionais da área, ressaltando que quanto maior o total arrecadado maior os valores dos impostos a serem pagos.

Observou-se então que cobrar um valor de 5 a 10 vezes maior que o valor do custo apresenta uma margem de conforto em relação ao lucro, visando que o capital de giro permaneça intacto, além do fato que a empresa deve manter em caixa dinheiro para imprevistos, tais como troca de equipamentos e instrumentais, indenização de funcionário e cotas extras caso necessário.

\section{CONSIDERAÇÕES FINAIS}

A capacidade de gerir é uma habilidade imprescindível para o sucesso comercial e financeiro de uma empresa. Entre os cirurgiões-dentistas, poucos profissionais tiveram a oportunidade de estudar o assunto na graduação ou pós-graduação. É essencial que os curso de Graduação em Odontologia assumam postura mais ativa sobre esse assunto na formação dos novos profissionais. Isso irá fortalecer a classe e, consequentemente, melhorará a qualidade dos serviços prestados.

\section{REFERÊNCIAS}

1. Andrade JM, Santana LMD, Gnoatto N. Odontologia e empreendedorismo: o perfil do cliente [Acesso em 2 fev 2014]. Disponível em: http://www.slideshare.net/thiagodant3/ar tigo-odontologia-e-empreendedorismoo-perfil-do-cliente

2. Arcier NM, Silva MM, Arcieri RM, Garbin CAS. A importância do marketing odontológico para enfrentar um mercado competitivo. Rev Odontol Araç. 2008;29(1):13-9.

3. Bezinelli L, Ramos M. Curso de Educação Continuada em Gestão de Consultório [Acesso em 21 fev 2014]. Disponível em:http://www.colgateprofissional.com. br/dentistas/Curso_ControleFinanceiro/d etalhes.

4. Brasil. Ministério da Educação e Cultura [home page na internet]. Perguntas frequentes sobre Educação Superior [acesso em 4 nov 2012]. Disponível em:

http://www.luizrosa.edu.br/fatec/faculda de/informativos/1\%203\%2010/document os/PERGUNTAS_RESPOSTAS.pdf.

5. Cordeiro JVBM, Ribeiro RV. Gestão de empresa [Acesso em 1 fev 2014]. Disponível em:

http://www.unifae.br/publicacoes/pdf/em presarial/1.pdf.

6. Dornelas JCA. Empreendedorismo corporativo: como ser empreendedor, inovar e se diferenciar na sua empresa. 2. ed. Rio de Janeiro: Elsevier; 2009.

7. Matsubara SM. Diretrizes estratégicas para uma clínica odontológica [monografia]. São Paulo (SP): Escola Politécnica da Universidade de São Paulo; 2009.

8. Oliveira L, Santos V, Gnoatto N. Odontologia e empreendedorismo: comportamento do profissional empreendedor [Acesso em 1 fev 2014]. Disponível em: http://www.slideshare.net/thiagodant3/ar tigo-odontologia-e-empreendedorismocomportamento-do-profissionalempreendedor

9. Pardini DJ, Santos RV. Empreendedorismo e interdisciplinaridade: uma proposta metodológica no ensino de graduação. Rev Administr FEAD-Minas 2008;5:157-72.

10. Price CT. Implementação do Planejamento Estratégico: Estudo de Caso de 
Consultório Odontológico [monografia]. Porto Alegre (RS): Universidade Federal do Rio Grande do Sul; 2009.

11. Sebrae. Planejamento Empresarial - Clínica Odontológica [Acesso em 12 fev 2014]. Disponível em: http://www.sebrae.com.br/bis/bds/BDS.n sf/87AEAD2B29654B0E832578A3004 BAE75/File/Clinicaodontologica.pdf.

12. Teixeira FFRA. A Influência do Sistema de Redes de Relações Sociais no Exercício de Empreender em Serviços Odontológicos [dissertação]. Belo Horizonte (MG): Universidade de FUMEC, Faculdade de Administração e Ciências Econômicas; 2009.

13. Zimbres RA. Efeitos da administração planejada em um consultório odontológico [Acesso em 12 fev 2014]. Anais Eletrônicos do III Congresso Internacional de Odontologia de Santa Catarina, 2006. Disponível em:

http://www.dr3.com.br/saude/Efeitos_ad m.pdf.

14. Zimbres RA. Marketing Odontológico ou Gestão Odontológica? [Acesso em 4 fev 2014]. Disponível em:

http://www.odontosites.com.br/odonto/g estao/ marketing-odontologico-ougestao-odontologica.html

15. Zuchini ARB, Lolli MCGS, Lolli LF, Lolli HA. Perfil profissional do cirurgião-dentista em associação ao conhecimento e utilização de marketing. Arq Odontol. 2012; 48(1):19-25.

\section{ABSTRACT \\ Dental clinic: management simulation of a dental clinic in an undergraduate dental school}

The constant influx of dentists into the market leads to an increase in competition among specialists. In this scenario, it is necessary to stand out by having points of difference, such as business management skills. Knowing how to manage an office or practice is a prerequisite for achieving stability and success in the profession. Nonetheless, most dental schools do not address this subject in their academic curriculum. Thus, the aim of this paper is to discuss the study of business management of a dental clinic in a dental school. The experience of managing a hypothetical company was under the direction of the "Class of 2013-2 Professor Antístenes Albernaz Alves Neto" of the Bahian School of Medicine and Public Health (EBMSP). This course work was part of the curriculum of Integrated Clinic V. A structural framework comprised of a President, two Superintendents and four Directors (Technical, Administrative, Financial and Scientific) was created as well as a fictitious currency (Bahianos- BH\$). During the three months of operation 2,180 procedures were performed (general and specialty procedures in Periodontics, Prosthodontics, Endodontics and Operative Dentistry). The total revenue collected from all procedures in the clinic was BH\$ 76,683.71, and the profit was BH\$ $38,341.85$. It is essential that dental schools take a proactive role in business management when it comes to training new professionals. Business strategy together with a welldefined operational and financial plan will influence the success of the owner as well as the company.

Descriptors: Dentistry. Practice Management. Dental. Job Market.

Correspondência para:

Renato Magalhães Costa

e-mail: renatomagalhaesc@hotmail.com

Rua Antônio Pedro, 422/201

Centro 48903-660 - Juazeiro/BA 